Trauma Berufskrankh 2006 - 8[Suppl 2]: S136-S142 DOI 10.1007/s10039-005-1018-6

Online publiziert: 1. September 2005

๑) Springer Medizin Verlag 2005

R. Winkel · M. Schlageter · BG-Unfallklinik, Frankfurt

\title{
Schmerzhaftes distales radioulnares Gelenk (DRUG)
}

\author{
Gibt es die Lösung?
}

ständnis dieses Gelenks geholfen haben. Diese sind schlagwortartig:

1. Der Ellenkopf ist das „Fundament" des Handgelenks [14].

2. Der Diskus mit seinen randständigen Verstärkungen der radioulnaren Bänder ist das „Ankerband“ [17], das die breite Speichenbasis mit der

Speiche und Hand in einem zum Körper gerichteten Halbkreis gestattet nicht nur das Greifen in alle Richtungen dieses Wendehalbkreises, sondern auch die Betrachtung beider Seiten einer Medaille oder sie befähigt uns, eine neue Seite aufzuschlagen (• Abb. 1).

Schmerzhaft werden kann das entwicklungsgeschichtlich junge Speichenwendegelenk durch seine frühe Alterung [23], nach Verletzungen oder durch Krankheit. Als Unfall- und Handchirurgen beschäftigen uns vorwiegend die häufigen Verletzungsfolgen und ihre Abgrenzung von Verschleißschäden sowohl bei der Behandlung als auch bei der Begutachtung. Die krankhaften entzündlichen, insbesondere rheumatischen Veränderungen berücksichtigen wir deshalb in diesem Zusammenhang nicht und verweisen auf die Literatur [2].

Die folgenden Ausführungen sollen durch die Deutung der Anatomie, die Berücksichtigung der Pathologie dieses Gelenks und der eigenen Erfahrungen aus der Behandlung von Verletzungsfolgen die Thesen erläutern, die uns zum Ver-
Hand wie einen Türflügel am Angelpunkt des feststehenden Ellenkopfs hält.

3. Brüche des Unterarms sind als „Gelenkbrüche“ [33] zu verstehen. Auch wenn die einander zugewandten Gelenkflächen von Speiche und Elle im körpernahen und körperfernen Speichen-Ellen-Gelenk nicht selbst betrof-

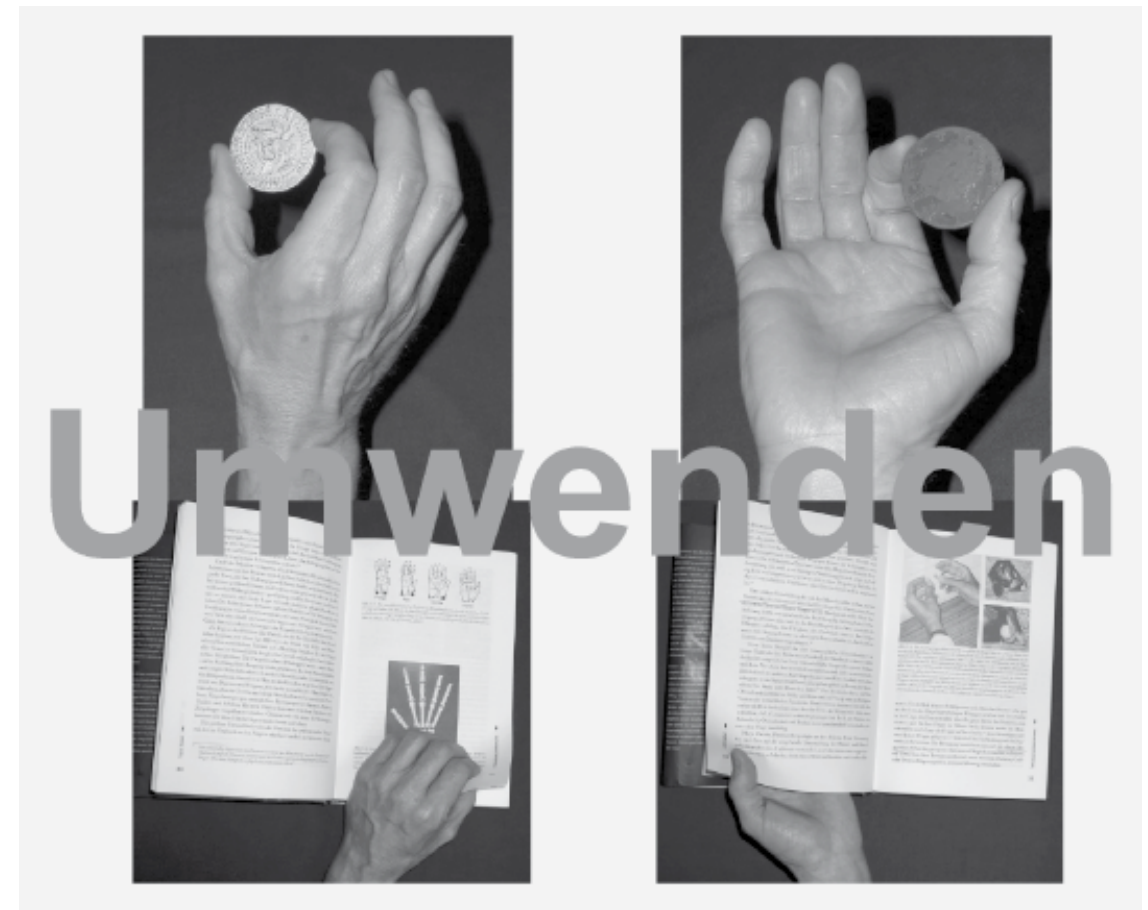

Abb. $1 \Delta$ Umwendung von Speiche und Hand gestattet die Betrachtung beider Seiten einer Medaille und das Aufschlagen einer neuen Seite 
fen sind, haben Dislokationen von Fragmenten der Metaphysen und/ oder Diaphysen von Radius und/oder Ulna durch den Verlust der Passform in den Speichen-Ellen-Gelenken dennoch Auswirkungen $[28,29,34]$ auf die Umwendbarkeit der Speiche.

4. Schäden im distalen Radioulnargelenk werden sehr viel schlechter toleriert als im Radiokarpalgelenk.

\section{Anatomie}

Erstaunlich und verwirrend sind die Beharrlichkeit, mit der wider besseres Wissen, dem scheinbaren Augenschein und dem allgemeinen Sprachgebrauch folgend, bei Verrenkungen im körperfernen Speichen-Ellen-Gelenk der feststehende Teil als der luxierende Teil bezeichnet wird [12, 17, 21], und die Ungenauigkeit, mit der von der Umwendung oder Drehung des Unterarms gesprochen wird, obwohl doch nur die Speiche sich dreht, wendet und ausrenkt $[11,26]$.

Die Hand ist mit der Speiche der Elle drehbar angelagert. Bei der Wendung der Hand dreht sich im körpernahen Teil des Doppelgelenks der Speichenkopf neben der Ellenbasis, gehalten durch das Ringband, um seine Achse. Im körperfernen Wendegelenk schwingt die Speichenbasis um den kleinen Ellenkopf, gehalten durch den Dreieckknorpel bzw. seine randständigen bandartigen Verstärkungen, die durch weitere Strukturen außerhalb des Gelenks unterstützt werden [26]. Seiner Wirkung nach könnte man das körpernahe Speichen-Ellen-Gelenk auch das „Drehgelenk der Speiche“ und das körperferne Speichen-Ellen-Gelenk das „Wendegelenk der Speiche" nennen.

Welches der radioulnaren Bänder in der Einwärts- und welches in der Auswärtsdrehung der Speiche gespannt ist, wird kontrovers diskutiert [3]. Nach Henschen [17], Schuind et al. [30] und Ward et al. [32] wird das dorsale radioulnare Band bei der Pronation und das palmare bei der Supination gestrafft.

Instabilitäten. Im distalen Radioulnargelenk sind sie Folge von Zerreißungen der randständigen Bänder des Dreieckknorpels, der Bänder zwischen Ellenkopf und Mondbein bzw. Dreieckbein und Mem- brana interossea [3]. Unfallbedingte Zerreißungen des Diskus sind nur bei gleichzeitiger Verletzung der radioulnaren Bänder möglich. Eine isolierte „Diskusruptur“ ohne Begleitverletzungen ist wahrscheinlich keine Unfallfolge [16].

Luxationen und Brüche. Im distalen Radioulnargelenk sind sie Verrenkungen der breiten Speichenbasis mit der Hand fort von dem kleinen, fest stehenden Ellenkopf. Besondere Formen sind der nach Galeazzi [10] benannte Bruch an der Grenze zum körperfernen Speichendrittel mit Verrenkung der Speichenbasis aus dem DRUG und die von Essex-Lopresti [5] beschriebene Verschiebung der Speiche zum Körper nach Bruch des Speichenkopfs mit Zerreißung sowohl der Membrana interossea als auch der radioulnaren Bänder und des Diskus.

Häufiger sind Beteiligungen des DRUG bei Brüchen der körperfernen Speiche, entweder in Form von Brüchen der Gelenkfläche der Speiche selbst gegenüber dem Ellenkopf oder in Form von Verdrehungen oder Verkippungen oder Verkürzungen der Speichenbasis [3, 22, 28]. Die hierdurch verursachten Inkongruenzen im DRUG sind radiologisch weniger augenfällig als die Brüche der Speiche, werden auf Dauer jedoch meist weniger gut ertragen als die Bruchfolgen im Radiokarpalgelenk.

Brüche des Griffelfortsatzes der Elle sind zu verstehen als Ausrisse des Ankerbands der Speichenbasis am Ellenkopf [26].

Pathologie des DRUG. $\mathrm{Zu}$ deren Verständnis haben maßgeblich Untersuchungen von Mikic [23] beigetragen. Der Verschleiß der dreieckförmigen Faserknorpelplatte beginnt schon in der 3. Dekade, und nach der 5. Dekade gibt es kaum noch einen normalen Diskus. Bei der Wendung der Speichenbasis mit der Hand um den fest stehenden Ellenkopf wirkt dieser wie ein „Bohrkopf“ auf die Faserknorpelscheibe und reibt sie auf. Ist der Diskus erst perforiert, wirkt die schädigende, drehende Reibung bei gleichzeitigem Druck des Ellenkopfs auf den gegenüber liegenden Teil des Mondbeins und das Band zwischen Mondbein und Dreieckbein, sodass letztlich das gesamte ellenseitige Handgelenk
Trauma Berufskrankh 2006

8[Suppl 2]: S136-S142

DOI 10.1007/s10039-005-1018-6

○) Springer Medizin Verlag 2005

R. Winkel · M. Schlageter

\section{Schmerzhaftes distales radioulnares Gelenk (DRUG).} Gibt es die Lösung?

\section{Zusammenfassung}

Die „Lösungen" für ein schmerzhaftes körperfernes Speichenellengelenk richten sich nach dem Schaden: die primäre oder sekundäre Wiederherstellung der Passform des Gelenks und/oder der radioulnaren Bänder, die Entfernung zerstörter zentraler Anteile des Diskus, die Druckentlastung durch Kürzung der Elle und bei Zerstörung des distalen Radioulnargelenks die den Schmerz lindernden und die Gebrauchsfähigkeit bessernden „Rettungsoperationen“ der Arthrodese mit Segmentresektion aus der Ulna, der Arthroplastik durch Teilentfernung des Ellenkopfs oder des Einsatzes einer Ellenkopfprothese.

\section{Schlüsselwörter}

DRUG · Distales Radioulnargelenk . Schmerzen · Passform des Gelenks . Ellenkopfprothese

\section{Pain in the distal radioulnar joint (DRUJ). Is there one solution?}

\section{Abstract}

According to the theses listed in the introduction, the "solutions" for pain in the distal radioulnar joint (DRUJ) depend on the kind of damage: reconstruction of the joint and or the radioulnar ligaments, resection of the central part of the discus, elimination of an ulnar impaction syndrome by wafer procedure or ulnar shortening, and, in case of destruction of the DRUJ, salvage procedures such as arthrodesis of the DRUJ with ulnar segment resection, hemiresection-interposition arthroplasty or implantation of an ulnar head prosthesis.

\section{Keywords}

DRUJ · Distal radio ulnar joint · Pain .

Diagnosis · Surgical therapy 


\section{Handchirurgie}
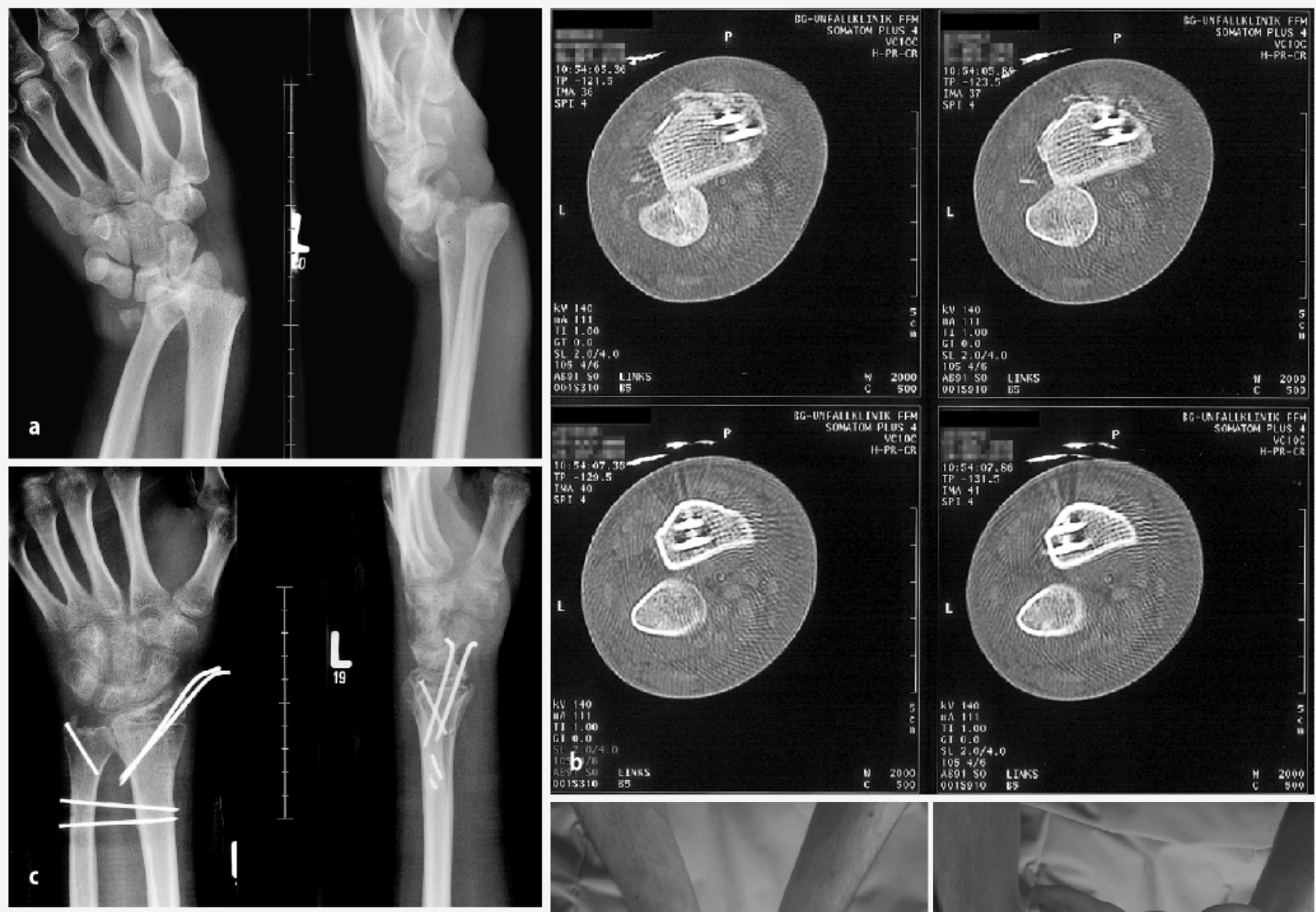

Abb. 2a-d $\Delta$ Fixierte Luxation im DRUG bei Bruch der körperfernen Speiche. a Röntgen d.-p. und seitlich, b CT in Querschnitten durch das DRUG, c Röntgen d.-p. und seitlich nach Einrichtung, Osteosynthese des Radius mit 2 Bohrdrähten, des Processus styloideus mit einer Schraube und Transfixation mit 2 Bohrdrähten, d nach Heilung freie Beweglichkeit
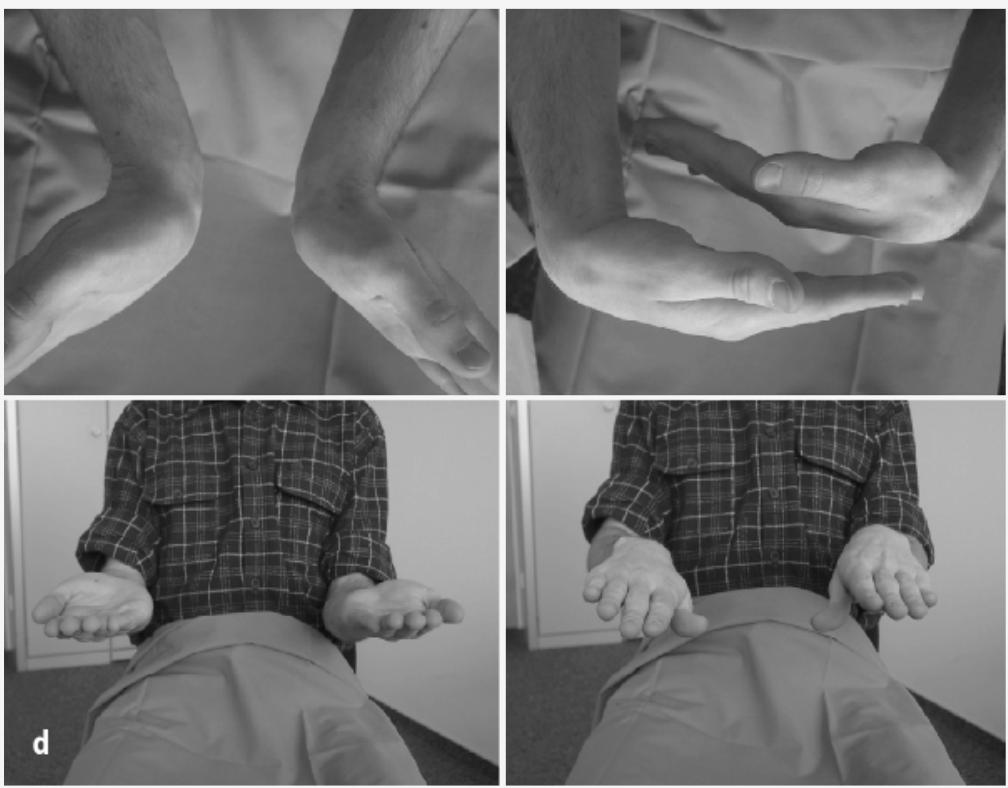

zerstört werden kann, bei erhaltenem radialseitigem Handgelenk.

\section{Diagnostik}

\section{Vorgeschichte}

Die altersbedingt zu erwartenden Schäden des Diskus sind bei allen Bewertungen von Befunden an einem schmerzhaften DRUG zu erinnern.
Die Vorgeschichte gibt Aufschluss über die beruflichen und privaten Belastungen der Hände, die Seite der Gebrauchshand und über die Art der Beschwerden seit ihrem Beginn, plötzlich oder schleichend, und über Verletzungen und deren Hergang.

Häufige Umwendebewegungen können bei einer zu langen Elle den Diskus aufreiben und Symptome eines Druckschadens, das so genannte ulnare Impac- tion- oder Abutment- oder Loading-Syndrom [9] auslösen.

Seltener als der Fall auf den Daumenballen ist der auf den Kleinfingerballen mit einwärts gedrehtem Unterarm und zwangsweiser handrückenwärtiger Überstreckung und Einwärtsdrehung. Dabei werden das Band zwischen Mond- und Dreieckbein und die Bänder zwischen Ellenkopf und diesen Handwurzelknochen verletzt [3]. 

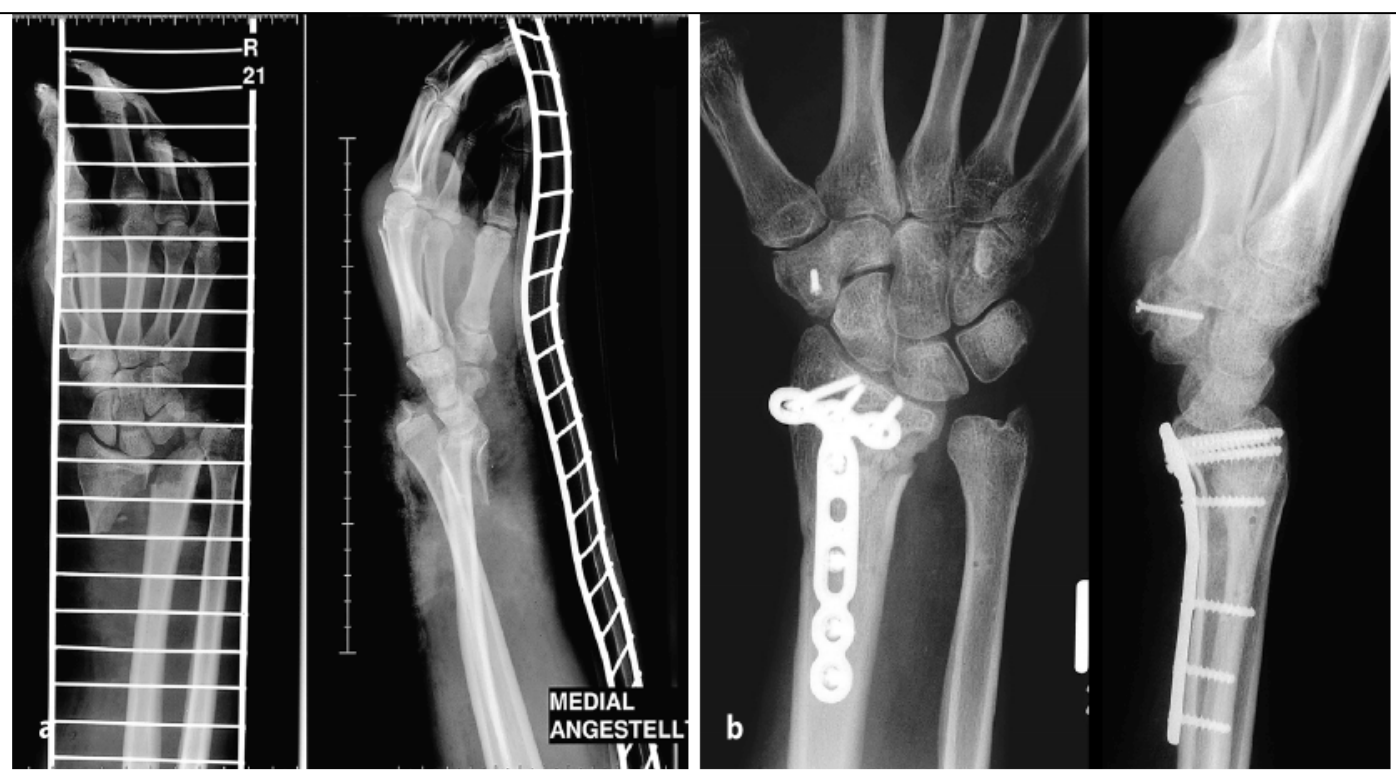

Abb. 3a, $b<$ Bruch und Verrenkung der Speichenbasis, nach Galeazzi [10] benannt. a Röntgen d.-p. und seitlich präoperativ, b Röntgen d.-p. und seitlich nach Plattenosteosynthese der Speiche von palmar

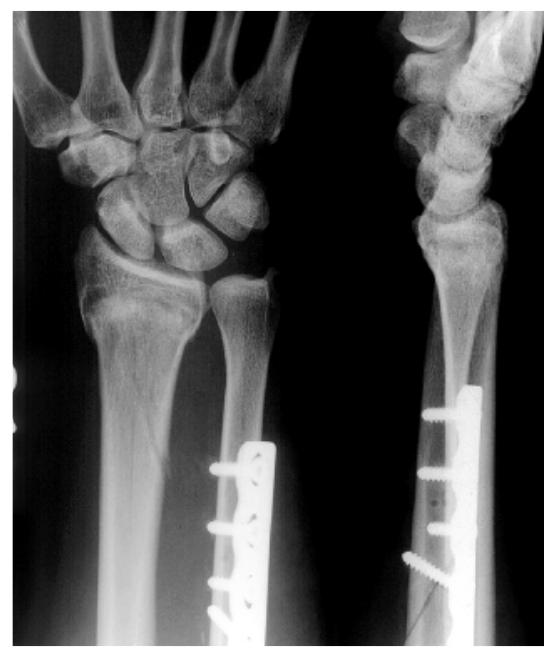

Abb. 4 A Röntgen d.-p. und seitl. nach Kürzung der Elle im Schaft um $\mathbf{3} \mathbf{~ m m}$ durch schräge Osteotomie und Osteosynthese mit Kompressionsplatte und Zugschraube

Eine Luxation der ECU-Sehne wird hervorgerufen durch eine zwangsweise Außendrehung mit gleichzeitiger Kantung der Hand zur Ellenseite [3].

In Anlehnung an Henschen [17] kann die Speichenbasis verrenken vom fest stehenden Ellenkopf fort zur Beugeseite

1. durch eine gewaltsame Beugung der Hand hohlhandwärts,

2. durch eine gewaltsame Einwärtsdrehung von Unterarm und Hand, ggf. verbunden mit einer zusätzlichen Beugung der Hand hohlhandwärts und

3. durch eine Auswärtsdrehung der Hand bei festgehaltenem Unterarm oder durch Einwärtsdrehung der Speiche bei festgehaltener Hand.

Zum Ausrenken der Speichenbasis vom Ellenkopf fort zur Streckseite können führen:

1. eine gewaltsame handrückenwärtige Streckung der Hand,

2. eine gewaltsame Auswärtsdrehung von Speiche und Hand, ggf. verbunden mit einer handrückenwärtigen $\mathrm{He}$ bung der Hand im Handgelenk und

3. eine Einwärtsdrehung der Hand bei festgehaltener Speiche und eine Auswärtsdrehung der Speiche bei festgehaltener Hand.

\section{Körperliche Untersuchung}

Der gesamte Unterarm vom Ellenbogen bis zur Hand wird betrachtet [34], und Änderungen der Form und des Umfangs, Fehlstellungen, z. B. Verschiebungen der Handwurzel mit der Speiche gegenüber dem Ellenkopf, Zeichen einer Störung der Gewebeernährung oder des Blutumlaufs, Minderung der Handbinnenmuskulatur, insbesondere am Kleinfingerballen, und die Verteilung der Gebrauchsspuren an den Händen werden festgestellt.

Die betastende Untersuchung erfolgt gleichzeitig und beidseitig an den entsprechenden Stellen. Schmerzen an der Ellenseite der Hand bei der gewaltsamen Kantung der Hand zur Ellenseite, verstärkt durch eine Auswärts- und/oder Einwärtsdrehung, deuten auf einen Schaden des
Discus triangularis bzw. ein so genanntes Ulna-Impaction-Syndrom hin [9].

Der einseitige Druckschmerz über der Sehne des ECU oder am Ansatz der FCUSehne, streckseitig über dem DRUG, dem Diskus, dem L-T-Band, dem Triquetrum oder dem ulnaren Recessus des Ulnokarpalgelenks [15] gibt den wesentlichen Hinweis auf den Ort des Schadens, der den Schmerz verursacht. Der einseitige Schmerz beim Versuch, die Speiche gegenüber dem Ellenkopf zur Beuge- oder Streckseite zu verschieben, und die Feststellung einer übernormalen Beweglichkeit sind Zeichen für einen Schaden der distalen radioulnaren Bänder, welche als „federnde Elle“ oder „Klaviertastenphänomen“ bezeichnet wurden [21].

Inkongruenzen im DRUG verursachen Schmerzen beim Druck der Speichenbasis gegen den Ellenkopf und lassen eine Drehbewegung bei gleichzeitiger Kompression nicht $\mathrm{zu}$ [9].

Ein isolierter Druckschmerz, meist palmar des Griffelfortsatzes der Elle, deutet auf eine Synovialitis im Recessus ulnaris hin $[15,27,26]$.

Die diagnostische Lokalanästhesie am vermuteten Ort der Schmerzauslösung kann zusätzlich hilfreich zur Lokalisierung sein [3]. Nur wenn der Ort der geklagten Beschwerden, die Stelle, die bei der körperlichen Untersuchung schmerzt, und eine Veränderung im Befund eines Bild gebenden Verfahrens übereinstimmen, hat diese Veränderung Krankheitswert. 


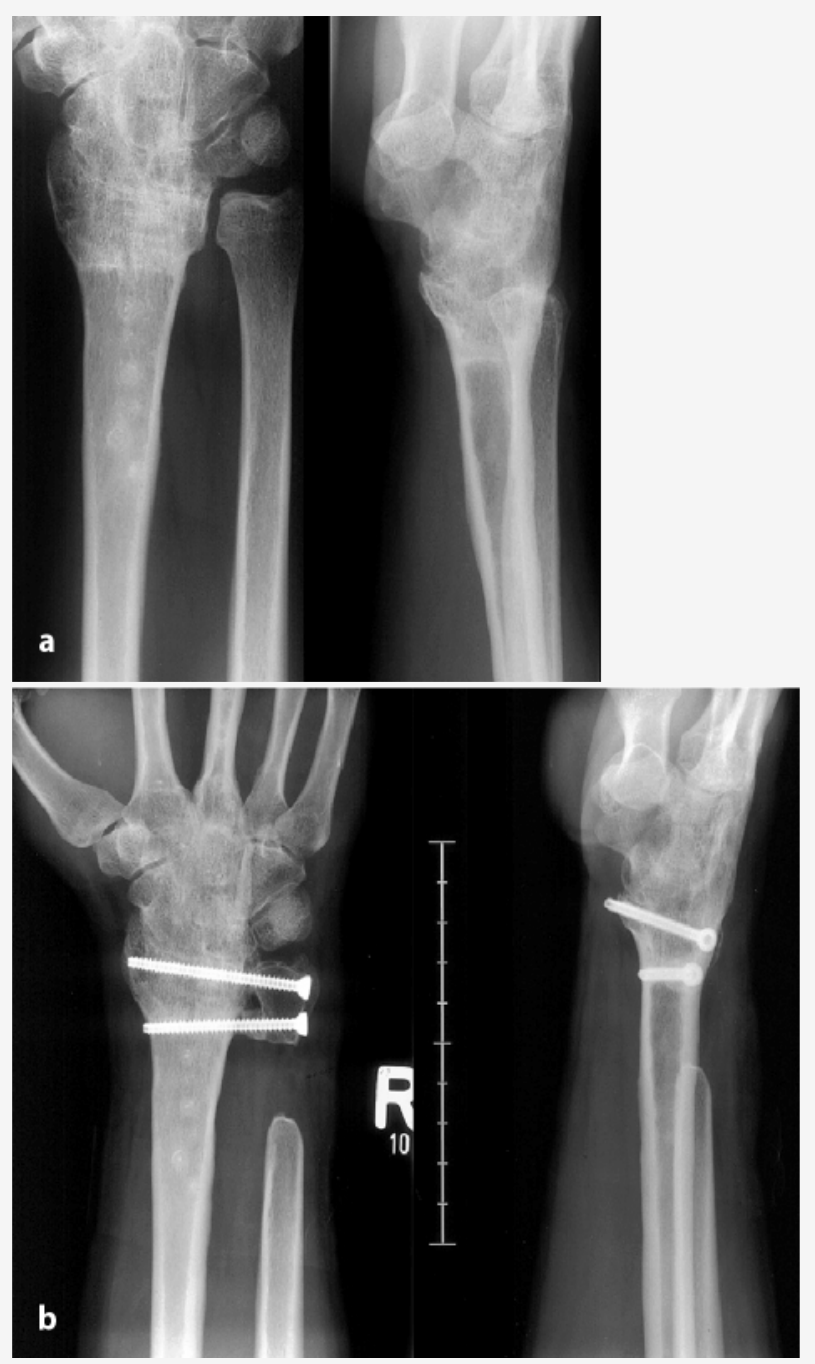

Abb. $5 a, b<$ Palliative Arthrodese des DRUG mit Segmentresektion aus der Elle nach Sauvé u. Kapandji [25]. a Röntgen d.-p. und seitl. nach Arthrodese des Handgelenkes, b Röntgen d.-p. und seitl. nach Heilung des Eingriffes nach SauvéKapandji

\section{Radiologische Diagnostik}

Sie wurde von Frahm [8] und Schmitt u. Lanz [27] für die Verletzung des DRUG übersichtlich dargestellt.

Die Röntgenuntersuchung. ist die wichtigste und fürs erste genügende Bild gebende Untersuchung in Neutralstellung der Handgelenke $[3,8,27]$. Die Haltung der Elle und die Drehstellung der Speiche können erkannt werden an der Projektion des Griffelfortsatzes der Elle in Beziehung zur Projektion des Ellenschafts und an der des Radius. Der Griffelfortsatz der Elle ist streckseitig wie das Olekranon gelegen [3].

Die Durchleuchtungsuntersuchung grenzt dynamische Instabilitäten des ulnaren Carpus von denen des DRUG ab.
Computertomographie. Sie ist für die $\mathrm{Be}$ urteilung der Kongruenz im DRUG unentbehrlich.

Arthrographie. Sie lässt inkomplette Diskusrisse sicherer erkennen als die MRT [8].

Im normalen Handgelenkarthrogramm stellen sich der Recessus ulnaris („praestyloid recess“) [3] und seine Verbindung zum Gelenk zwischen Dreieckbein und Erbsenbein dar. Asymptomatisch können Risse des S-L Bandes (36\%), des L-T Bandes (40\%) und des Discus triangularis (50-60\%) sein. Diese Veränderungen beweisen nicht einen ursächlichen Zusammenhang zu einer Verletzung oder den abzuklärenden Beschwerden, weil sie oft symmetrisch vorkommen oder nicht mit dem Ort des Schmerzes bei der körperlichen Untersuchung übereinstimmen [3].

Die indirekte $M R$-Arthrographie hat eine hohe Sensitivität für Diskusläsionen und Begleitverletzungen und kann eine Screeningmethode zur Indikationsstellung für eine therapeutische Arthroskopie werden.

Arthroskopie. Sie hat ihren festen Platz in der Diagnostik und Behandlung von Verletzungen oder Schäden des Diskus $[3,6$, $16,31]$.

Grundlage der Einteilung der Schäden des Discus triangularis ist die Klassifikation nach Palmer [24].

\section{Therapie}

Die Behandlung von Schmerzen in und um das DRUG gründet auf der Diagnose, d. h. dem Ort und der Art des Schadens, der die Schmerzen verursacht. Gewarnt sei vor jedem Entschluss zu einer eingreifenden Behandlung, der nur auf einer $\mathrm{Pa}$ thologie in einem Bild gebenden Verfahren beruht - meist bringen die Patienten nur Filme einer MRT-Untersuchung mit, ohne Vorlage von Röntgenaufnahmen des Handgelenks in Neutralstellung. Nur eine Übereinstimmung des Ortes der Schmerzen mit dem Befund einer körperlichen Untersuchung und der Veränderung im MRT begründet die Anzeige zu einem ärztlichen Eingriff.

Häufiger als Brüche der Gelenkflächen des DRUG - seien es Brüche des Ellenausschnitts der Speiche (Incisura radii) oder Brüche des Ellenkopfs - führen Brüche der körperfernen Speiche, die eine Kippung und Drehung der Speichengelenkfläche zur Handwurzel und somit eine Verschiebung der Incisura radii zur Folge hatten [34], zu Schmerzen im DRUG und ulnokarpalen Gelenk. Die Abrissbrüche des Griffelfortsatzes der Elle sind als knöcherne Abrisse des Diskus zu betrachten und können zur Instabilität im DRUG führen, wenn der Bruchspalt durch die Basis des Processus styloideus ulnae verläuft [3].

Die Heilung eines Bruchs der körperfernen Speiche mit einer Knickbildung von nur $10^{\circ}$ zur Streckseite kann die Umwendbewegung behindern [18]. Deshalb sollen Korrekturosteotomien an der ehemaligen Bruchstelle die Passform des Gelenks wiederherstellen.

Aus der eingangs aufgestellten These [33], dass Brüche der Unterarmknochen wegen der Speichen-Ellen-Gelenke als Ge- 
lenkbrüche zu betrachten sind, leitet sich folgerichtig die Forderung nach der anatomischen Einrichtung und inneren Befestigung ab [28, 29, 33, 34].

Als Beispiel möge der Fall einer fixierten Luxation im DRUG dienen (• Abb. 2). Die schon im seitlichen Strahlengang erkennbare Luxation wurde durch das CT bestätigt. Die vom Koautor durchgeführte offene Einrichtung der Luxation nach vorangegangener Osteosynthese der Radiusfraktur mit Bohrdrähten, die Transfixation des Radius in der reponierten Stellung gegen die Ulna und die Reposition und Osteosynthese des Processus styloideus ulnae führten zur Wiederherstellung einer freien Beweglichkeit.

Auch die nach Galeazzi [10] benannte Verletzung wurde vom Koautor offen eingerichtet und mit einer Platte und Schrauben von der Beugeseite befestigt. Die Umwendebeweglichkeit zum Zeitpunkt der ersten Rentenbegutachtung betrug für Pro- und Supination $70 / 0 / 60^{\circ}$ (• Abb. 3).

Der Discus triangularis wird bei einer Verletzung im horizontalen Zentrum und bei verschleißbedingten zentralen Schäden débridiert bzw. teilreseziert $[16,24]$. Im Vortrag zeigte ich die durch den Coautor durchgeführte arthroskopische Resektion des zerstörten zentralen Teiles des Discus über einer zu langen Elle, die im selben Eingriff gekürzt wurde (• Abb. 4). Ulnare Abrissverletzungen können bei erhaltener Stabilität des DRUG genäht werden $[16,31]$.

Für die Begutachtung isolierter Diskusschäden ist die Feststellung von Hempfling [16] wichtig, dass eine Diskusruptur nur dann eintreten kann, wenn auch eine Verletzung der radioulnaren Bänder vorliegt.

Der aufreibende Druck durch den Ellenkopf auf den sich darüber drehenden Diskus und den ellenseitigen Teil der Handwurzel kann vermindert werden durch die Entfernung einer Scheibe aus dem Ellenkopf radialseitig des Ansatzes der radioulnaren Bänder ([7] und Skizze (Abb. 30-33) in [3]), oder durch die Kürzung der Elle im Schaft [9, 20] (• Abb. 4). Der dem Mondbein gegenüber liegende Teil des Ellenkopfs kann entweder von außen streckseitig körpernah der radioulnaren Bänder oder arthroskopisch nach Entfernung des mittleren Teils des Diskus bis zu 4 mm gekürzt werden [7]. Die Kürzung der Elle im Schaft kann durch eine quere oder stufenförmige oder schräge Osteotomie erfolgen $[3,9,20]$ (• Abb. 4).

Die schräge Trennung kann mit 2 oder 3 zusammen eingespannten Sägeblättern mit einem Schnitt [20] durchgeführt werden. Die Osteosynthese erfolgt mit einer Kompressionsplatte und einer Zugschraube $[9,20]$ (• Abb. 4).

Bei der Essex-Lopresti-Verletzung [5] kann eine Kürzung der Elle nur dann bleibenden Erfolg haben, wenn die Narbe der Membrana interossea ausreichend fest ist.

Obwohl die radioulnaren Bänder das „Ankerband“ der Speichenbasis am Ellenkopf sind, wird eine wesentliche Instabilität im DRUG nach Riss dieser Bänder erst bei einer zusätzlichen Verletzung der ulnokarpalen Bänder, der Membrana interossea, dem dorsokarpalen Bandkomplex und dem Retinaculum extensorum auftreten und kann dann noch durch die dynamischen Kräfte des ECU und des Pronator quadratus kompensiert werden [3].

Alle Versuche, die distalen radioulnaren Bänder körpernah des distalen Radioulnargelenks zu ersetzen, sind gescheitert. $\mathrm{Ob}$ die Versuche der anatomischen Rekonstruktion erfolgreicher sind, bleibt abzuwarten $([1,4]$ und Skizze (Abb. 27-32) in [3]).

Ist das distale Radioulargelenk zerstört, können die palliativen „Rettungsoperationen“ der Arthroplastik durch Teilentfernung des Ellenkopfs [3] oder der Arthrodese des DRUG und Segmentresektion der Elle $[13,18,25,28]$ eine schmerzarme und minderbelastbare Umwendbeweglichkeit der Speiche wiederherstellen. Auch uns hat sich der Eingriff nach Sauvé u. Kapandji [25] bewährt (• Abb. 5). In diesem Fall waren nach Arthrodese des Handgelenkes unerträgliche Schmerzen beim Wenden der Hand verblieben. Nach Heilung der zusätzlichen Arthrodese des DRUG und Segmentresektion aus der Elle war die Auswärtsdrehung von $60^{\circ}$ auf $80^{\circ}$ gebessert und die Schmerzen gelindert bei einer Wendbarkeit der Speiche nach außen/ innen um $80^{\circ} \% / 80^{\circ}$.

Der Einsatz einer Ellenkopfprothese bleibt Einzelfällen vorbehalten [29].

\section{Fazit für die Praxis}

- Die breite Speichenbasis wendet sich mit der Hand um den kleinen Ellenkopf, das „Fundament" des Handgelenks, gehalten durch das „Ankerband“ der randständigen Verstärkungen des Diskus. Die Speichenbasis ist es, die luxiert, nicht der Ellenkopf.

- Brüche von Speiche und Elle wirken sich bei einer Dislocation auf die Paßform im DRUG und somit auf die Wendbarkeit der Speiche aus.

- Fehlstellungen im DRUG werden sehr viel schlechter toleriert als solche im Radiokarpalgelenk.

- Der Discus ist die Schwachstelle des entwicklungsgeschichtlich jungen DRUG. Er verschleißt früh, beginnend vom Ellenkopf zum Handgelenk fortschreitend.

- Eingriffe mit dem Ziel der Druckentlastung im Ulnokarpalgelenk werden voraussichtlich an Bedeutung zunehmen.

- Krankheitswert haben Veränderungen in Befunden Bild gebender Verfahren, meist des MRT, nur dann, wenn der Ort der Veränderung mit der Stelle der geklagten Beschwerden übereinstimmt und wenn eben da ein Schmerz bei der körperlichen Untersuchung ausgelöst wurde.

- Für die gutachtliche Praxis ist wichtig: Ein isolierter Schaden des Diskus ohne andere Schäden, die nachweisbar verletzungsbedingt sind, ist wahrscheinlich keine Unfallfolge.

\section{Korrespondierender Autor \\ Dr. R. Winkel}

BG-Unfallklinik,

Friedberger Landstraße 430,

60389 Frankfurt

E-Mail: www.buk.bibliothek.

ffm@t-online.de

\section{Danksagung}

Die Autoren danken Frau B. Kunze für das Schreiben des Manuskriptes.

Interessenkonflikt: Der korrespondierende Autor versichert, dass keine Verbindungen mit einer Firma, deren Produkt in dem Artikel genannt ist, oder einer Firma, die ein Konkurrenzprodukt vertreibt, bestehen. 


\section{Handchirurgie}

\section{Literatur}

1. Adams BD (2000) Anatomic reconstruction of the distal radioulnar ligaments for DRUJ instability. Techn Hand Upper Extremity Surg 4: 154-160

2. Borisch N, Haußmann P (2004) Das Caput-ulnaeSyndrom. Orthopäde 33: 692-697

3. Bowers WH (1999) The distal radioulnar joint. In: Green DP, Hotchiss RN, Pederson WC (eds) Green's operative hand surgery, 4th edn, vol 1. Churchill Livingstone, Edinburgh London New York, pp 9861032

4. Butz PC, Meyer VE (1995) Dynamic stabilizing reconstruction of the triangular fibrocartilage complex (TFCC). 6th Congress of the Internatonal Federation of Societies for Surgery of the Hand IFSSH, Helsinki, Monduzzi Editore S.p.A-Bologna, pp 567570

5. Essex-Loprestri P (1951) Fractures of the radial head with distal radio-ulnar dislocation. J Bone Joint Surg Br 33B: 244-247

6. Feldkamp G (2004) Die arthroskopische "wafer procedure" bei degenerativen Discus-ulnocarpalis-Rissen mit ulnokarpalem Kompressionssyndrom. Orthopäde 33: 685-691

7. Feldon P, Terrono AL, Belsky MR (1992) Wafer distal ulna resection for triangular fibrocartilage tears and/or ulnar impaction syndrome. J Hand Surg [Am] 17A: 731-737

8. Frahm R (2004) Bildgebende Diagnostik des ulnaren Handgelenkschmerzes. Orthopäde 33: 645656

9. Friedmann StL, Palmer AK (1991) The ulnar impaction syndrome. Hand Clin 7: 295-310

10. Galeazzi R (1935) Über ein besonderes Syndrom bei Verletzungen im Bereich der Unterarmknochen. Arch Orthop Unfallchir 35: 557-562

11. Gilula LA, Mann FA, Dobyns JH et al. (2002) Wrist: terminology and definitions. J Bone Joint Surg Am [Suppl 1] 84-A: 21

12. Haas HG (1988) Subluxationen und Luxationen der Ulna im distalen Radioulnargelenk. In: Buck Gramcko D, Nigst H (Hrsg) Bibliothek für Handchirurgie, Handgelenkverletzungen. Hippokrates, Stuttgart, S111-118

13. Haferkamp H (2000) Rekonstruktion des zerstörten Radioulnargelenkes, Rettungsoperationen. Osteosynthese Int [Suppl 1] 8: 83-85

14. Hagert CG (1994) Distal radius fracture and the distal radioulnar joint - anatomical considerations. Handchir Mikrochir Plast Chir 26: 22-26

15. Hahn P, Schmitt R (2000) Die Bursitis des Recessus ulnaris. Handchir Mikorchir Plast Chir 32: 375-378

16. Hempfling $\mathrm{H}$ (2004) Die Läsionen des Handgelenkdiskus. Orthopäde 33: 657-675

17. Henschen C (1938) Die Operation der Luxatio ulnae im unteren Radio-Ulnargelenk. Schweiz Med Wochenschr 18: 466-472

18. Hirahara H, Neale PG, Lin YT et al. (2003) Kinematic and torque-related effects of dorsally angulated distal radius fractures and the distal radial ulnar joint. J Hand Surg [Am] 28A: 614-621

19. Kapandji Al (1998) Amélioration technique da I' opération Kapandji-Sauvé, dite Technique III. Ann Chir Main 17: 78-86

20. Krimmer H, Schoonhoven J van, Tränkle $M$ et al. (1998) Ulna-Impaction Syndrom: Therapie durch druckentlastende Verfahren am Ulnakopf. Handchir Mikrochir Plast Chir 30: 370-374

21. Liebolt FL (1953) A new procedure for treatment of luxation of the distal end of the ulna. J Bone Joint Surg [Am] 35A: 261-262
22. Matthews LS, Kaufer H, Garver DF et al. (1982) The effect on supination-pronation of angular malalignment of fractures of both bones of the forearm. J Bone Joint Surg [Am] 64A,1: 14-17

23. Mikic ZDj (1978) Age changes in the triangular fibrocartilage of the wrist joint. J Anat 126: 367-384

24. Palmer AK (1990) Triangular fibrocartilagae disorders: injury patterns and treatment. Arthroscopy 6 : 125-132

25. Sauvé L, Kapandji Al (1936) Nouvelle technique de traitment chirurgical des luxations récidivantes isolées de l'extrémité inférieure du cubitus. J Chir 47: 589-594

26. Schmidt HM (2004) Die Anatomie des ulnokarpalen Komplexes. Orthopäde 33: 628-637

27. Schmitt R, Lanz U (1996) Bildgebende Diagnostik der Hand. Hippokrates, Stuttgart, S 128-148

28. Schoonhoven J van, Prommersberger KJ (2000) Differentialdiagnose und Therapie der posttraumatisch gestörten Unterarmdrehbewegung. Handchir Mikrochir Plast Chir 32: 390-398

29. Schoonhoven J van, Lanz U (2004) Rettungsoperationen und deren Differentialindikationen am distalen Radioulnargelenk. Orthopäde 33: 704-714

30. Schuind F, An KN, Berglund L et al. (1991) The distal radioulnar ligaments: a biomechanical study. J Hand Surg [Am] 16A: 1106-1114

31. Tünnerhoff HG, Haußmann P (2001) Wann ist die arthroskopische Refixation des Discus ulnocarpalis bei ulnarem Abriss indiziert? Handchir Mikrochir Plast Chir 33: 239-244

32. Ward LD, Ambrose CG, Masson MV et al. (2000) The role of the distal radioulonar ligaments, interosseous membrane and joint capsule in distal radioulnar joint stability. J Hand Surg [Am] 25A: 341351

33. Weinberg A (2005) 15. Ostdeutsches AO-Seminar, 11.-12.02.05, Görlitz

34. Williams CS, Jupiter JB (1993) Das schmerzhafte UInokarpalgelenk. Orthopäde 22: 36-45

35. Wilson FR (2000) Die Hand - Geniestreich der Evolution. Klett-Cotta, Stuttgart, S 32 